\title{
Analytical and Bioanalytical Chemistry, and the International Year of the Periodic Table
}

\author{
Adam T. Woolley ${ }^{1}$ \\ Published online: 5 September 2019 \\ (C) Springer-Verlag GmbH Germany, part of Springer Nature 2019
}

At the start of each semester when I teach advanced chemical analysis to undergraduate and graduate students, I affirm the central importance of analytical chemistry. One of the key evidences I use in support of the significance of analytical chemistry is the first paper published in Chemical Reviews [1]. The author of this inaugural Chem. Rev. paper was the first American Nobel Laureate in chemistry, Theodore William Richards, who received the Nobel Prize in 1915 for determination of highly accurate atomic masses of many of the elements. Richards achieved these elemental determinations with unprecedented accuracy through well-planned, careful gravimetric analysis. While Richards was measuring atomic masses gravimetrically, J. J. Thomson was building the first mass spectrometers, allowing him to discover neon's isotopes 20 and 22. Francis W. Aston, who studied under Thomson, subsequently used mass spectrometry to measure atomic masses of over 200 isotopes, for which he received the Nobel Prize in Chemistry in 1922. A century ago, both classical and emerging instrumental methods of analytical chemistry were key in determining atomic masses for the periodic table.

Furthermore, methods now within the domain of analytical chemistry, including optical spectroscopy, X-ray spectroscopy, and radiochemistry, were essential in placing elements into their correct columns/groups within the periodic table. So, not only is analytical chemistry responsible for the atomic masses reported in the periodic table, but it is also essential for the placement of elements within the periodic table.

Adam T. Woolley

woolley-abc-editor@byu.edu

1 Chemistry and Biochemistry Department, Brigham Young University, Provo, UT 84602-5700, USA
Notably, the periodic table continues to be an important factor in analytical and bioanalytical chemistry today. For instance, mass spectrometry measures mass-to-charge ratios, and once the charge is known, so is the mass. However, analysis of (for example) chlorine via ICP-MS doesn't yield the average (atomic) mass of the stable isotopes found in the periodic Table (35.453); instead, a larger peak at 35 (monoisotopic mass) and a smaller one at 37 (heavy isotope containing two additional neutrons) are in the mass spectrum. Thus, understanding the periodic table is still essential for today's analytical chemists. Indeed, an emerging bioanalytical application of these isotopic distributions is the technique of mass cytometry, wherein individual cells are tagged with highly multiplexed biochemical labels, each linked to a different elemental isotope [2]. Mass spectrometric analysis of the labeled cells then enables high-throughput determination of binding interactions, cell receptors, etc.

In this, the International Year of the Periodic Table [3], it is important to remember the longstanding and close connection of chemical analysis with the periodic table over its 150-year history. Building on that lengthy relationship, the fields of analytical and bioanalytical chemistry will continue their strong association with the periodic table in the decades that follow.
Adam T. Woolley
Chair Editor, ABC
woolley-abc-editor@byu.edu 


\section{References}

1. Richards TW. Atomic weights and isotopes. Chem Rev. 1924;1:140.

2. Mironov GG, Bouzekri A, Watson J, Loboda O, Ornatsky O, Berezovski MV. Aptamer-facilitated mass cytometry. Anal Bioanal Chem. 2018;410:3047-51.

3. Meija J. Elemental pub quiz challenge. Anal Bioanal Chem. 2019. https://doi.org/10.1007/s00216-019-02057-w.

Publisher's note Springer Nature remains neutral with regard to jurisdictional claims in published maps and institutional affiliations.

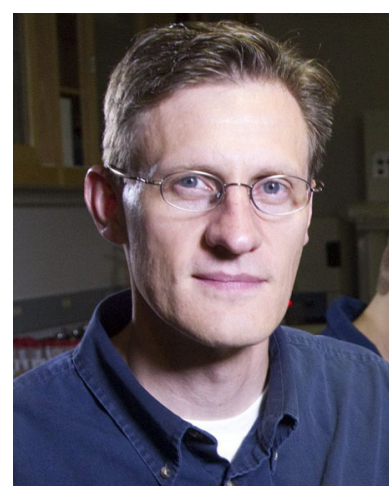

Adam T. Woolley is Chair Editor of Analytical and Bioanalytical Chemistry, and University Professor in the Department of Chemistry and Biochemistry Brigham Young University in Provo, Utah, USA. His current research focuses on 3D printed integrated microfluidics for biomarker measurement, analytical systems for the identification of bacteria and antibiotic resistance genes in sepsis, and biotemplated fabrication of nanoelectronics. 